\author{
ANNALS OF “DUNAREA DE JOS” UNIVERSITY OF GALATI \\ MATHEMATICS, PHYSICS, THEORETICAL MECHANICS \\ FASCICLE II, YEAR X (XLI) 2018, No. 2
}

Article DOI: https://doi.org/10.35219/ann-ugal-math-phys-mec.2018.2.04

\title{
A GENERAL FIXED POINT THEOREM FOR MAPPINGS SATISFYING AN IMPLICIT RELATION IN COMPLETE $G p$ - METRIC SPACES
}

\author{
Valeriu Popa ${ }^{1}$, Alina-Mihaela Patriciu ${ }^{2}$ \\ 1 "Vasile Alecsandri" University of Bacau, Romania \\ ${ }^{2 *}$ Faculty of Sciences, Department of Mathematics and Computer Sciences, "Dunarea de Jos" University of \\ Galati, Romania, e-mail: Alina.Patriciu@ugal.ro
}

\begin{abstract}
The purpose of this paper is to prove a general fixed point theorem in complete $G p$ - metric spaces for mappings satisfying a new type of implicit relation. If $G p$ - metric is symmetric, we prove that the fixed point problem is well posed.
\end{abstract}

Keywords: fixed point, $G p$ - metric space, well posedness of fixed point problem, implicit relation.

\section{INTRODUCTION}

In [13], [14], Dhage introduced a new class of generalized metric spaces, named $D$ - metric spaces. Mustafa and Sims [21], [22] proved that most of the claims concerning the fundamental topological structures on $D$ - metric spaces are incorrect and introduced an appropriate notion of generalized metric space, named $G$ - metric spaces. In fact, Mustafa, Sims and other authors [1], [17], [20] - [27], [38], [39] studied many fixed point results for self mappings in $G$ - metric spaces under certain conditions.

In 1994, Matthews [19] introduced the concept of partial metric space as a part of the study of denotional semantics of dataflows and proved the Banach contraction principle in such spaces. Recently, in [2], [7], [11], [15], [16] and in other papers, some fixed point theorems under various contractive conditions in partial metric spaces are proved.

Quite recently, Zand and Nezhad [45] introduced a generalization and unification of $G$ - metric space and partial metric space, named $G p$ - metric space. In [8], some fixed point theorems in $G p$ metric spaces are proved. Other results are obtained in [9] and [10].

The notion of well posedness of fixed point problem generated more interest to several mathematicians, for example [12], [18], [37].

Several classical fixed point theorems and common fixed point theorems have been unified considering a general condition by an implicit relation in [28], [29] and in other papers. Recently, the method is used in the study of fixed points in metric spaces, symmetric spaces, quasi - metric spaces, $b$ - metric spaces, ultra - metric spaces, Hilbert spaces, convex metric spaces, reflexive spaces, compact metric spaces, paracompact metric spaces, in two and three metric spaces, for single - valued mappings, hybrid pairs of mappings and set - valued mappings. Recently, the method has been used in the study of fixed points for mappings satisfying contractive/extensive conditions of integral type, in fuzzy metric spaces, probabilistic metric spaces and intuitionistic metric spaces. Also, the method allows the study of local and global properties of fixed point structures. 
The study of fixed points for mappings in $G$ - metric spaces satisfying implicit relations is initiated in [32] - [36] and in other papers. The study of fixed points for mappings satisfying an implicit relation in partial metric spaces is initiated in [44].

In [3] - [6], [30], [31] and in other papers, the authors studied well posedness of fixed point problem for mappings satisfying implicit relations.

The purpose of this paper is to prove a general fixed point theorem for mappings satisfying a new type of implicit relation in the $G p$ - metric spaces. We prove that for these mappings, if the $G p$ metric is symmetric, then the fixed point problem is well posed.

\section{PRELIMINARIES}

Definition 1 ([45]) Let $X$ be a nonempty set. A function $G: X^{3} \rightarrow \mathrm{R}_{+}$is called a Gp metric on $X$ if the following conditions are satisfied:

$\left(G p_{1}\right): x=y=z$ if $G p(x, y, z)=G p(x, x, x)=G p(y, y, y)=G p(z, z, z)$;

$\left(G p_{2}\right): 0 \leq G p(x, x, x) \leq G p(x, x, y) \leq G p(x, y, z)$ for all $x, y, z \in X$, with $y \neq z$;

$\left(G p_{3}\right): G p(x, y, z)=G p(y, z, x)=\ldots$ (symmetry in all three variables);

$\left(G p_{4}\right): G p(x, y, z) \leq G p(x, a, a)+G p(a, y, z)-G p(a, a, a)$ for all $x, y, z, a \in X$.

The pair $(X, G p)$ is called a $G p$ - metric space.

Definition 2 ([45]) Let $(X, G p)$ be a Gp - metric space and $\left\{x_{n}\right\}$ be a sequence in $X . A$ point $x \in X$ is said to be the limit of the sequence $\left\{x_{n}\right\}$ (we write $x_{n} \rightarrow x$ and we say that $\left\{x_{n}\right\}$ is $G p$ - convergent to $x)$ if $\lim _{m, n \rightarrow \infty} G p\left(x, x_{n}, x_{m}\right)=G p(x, x, x)$.

Theorem 3 ([8]) Let $(X, G p)$ be a Gp - metric space. Then, for any $\left\{x_{n}\right\} \in X$ and $x \in X$, the following conditions are equivalent:

a) $\quad\left\{x_{n}\right\}$ is $G p$ - convergent to $x$;

b) $\quad G p\left(x_{n}, x_{n}, x\right) \rightarrow G p(x, x, x)$ as $n \rightarrow \infty$;

c) $\quad G p\left(x_{n}, x, x\right) \rightarrow G p(x, x, x)$ as $n \rightarrow \infty$.

Definition 4 ([45]) Let (X,Gp) be a Gp - metric space.

1) A sequence $\left\{x_{n}\right\}$ of $X$ is called a Gp - Cauchy sequence if $\lim _{n, m \rightarrow \infty} G p\left(x_{n}, x_{m}, x_{m}\right)$ exists and is finite.

2) A Gp - metric space is said to be Gp - complete if and only if every Gp-Cauchy sequence in $X$ Gp - converges to $x \in X$ such that $\lim _{n, m \rightarrow \infty} G p\left(x_{n}, x_{m}, x_{m}\right)=G p(x, x, x)$.

Lemma 5 ([8]) Let $(X, G p)$ be a $G p$ - metric space.

1) If $G p(x, y, z)=0$, then $x=y=z$.

2) If $x \neq y$ then $G p(x, x, y)>0$.

Definition 6 ([45]) $A G p$ - metric on $X$ is said to be symmetric if $G p(x, y, y)=G p(y, x, x)$.

In this case, $(X, G p)$ is said to be symmetric.

Lemma 7 ([45]) Let $(X, G p)$ be a Gp-metric space. Then $G p(x, x, y) \leq 2 G p(x, y, y)$ for all $x, y \in X$.

Lemma 8 ([8]) Let $(X, G p)$ be a $G p$ - metric space and $\left\{x_{n}\right\}$ a sequence in $X$. Assume that $\left\{x_{n}\right\}$ is $G p$ - convergent to a point $x \in X$ with $G p(x, x, x)=0$. Then $\lim _{n \rightarrow \infty} G p\left(x_{n}, y, y\right)=G p(x, y, y)$ for all $y \in X$. Moreover, $\lim _{n, m \rightarrow \infty} G p\left(x_{n}, x_{m}, x\right)=0$.

Lemma 9 Let $(X, G p)$ be a Gp - metric space and $\left\{x_{n}\right\}$ is Gp - convergent to a point $x \in X$ with $G p(x, x, x)=0$. Then $\lim _{n \rightarrow \infty} G p\left(x_{n}, x_{n}, y\right)=G p(x, x, y)$ for all $y \in X$. 
Proof. By $\left(G p_{4}\right)$,

$$
G p(y, x, x) \leq G p\left(y, x_{n}, x_{n}\right)+G p\left(x_{n}, x, x\right) .
$$

Hence,

$$
G p(y, x, x)-G p\left(x_{n}, x, x\right) \leq G p\left(y, x_{n}, x_{n}\right) \leq G p(y, x, x)+G p\left(x, x_{n}, x_{n}\right) .
$$

Letting $n$ tends to infinity, by Theorem 3 we obtain

$$
G p(y, x, x) \leq \lim _{n \rightarrow \infty} G p\left(y, x_{n}, x_{n}\right) \leq G p(y, x, x) .
$$

Hence,

$$
\lim _{n \rightarrow \infty} G p\left(y, x_{n}, x_{n}\right)=G p(y, x, x)
$$

\section{IMPLICIT RELATIONS}

Let $\mathscr{F}_{G} p$ be the set of all continuous functions $F: \mathrm{R}_{+}^{6} \rightarrow \mathrm{R}$ satisfying

$\left(\mathrm{F}_{1}\right)$ : $\mathrm{F}$ is non - increasing in variables $t_{3}, t_{4}, t_{5}, t_{6}$;

$\left(\mathrm{F}_{2}\right)$ : There exists $h_{1} \in[0,1)$ such that for all $u, v \geq 0, F(u, v, v, u, u+v, u) \leq 0$ implies $u \leq h_{1} v$;

$\left(\mathrm{F}_{3}\right)$ : There exists $h_{2} \in[0,1)$ such that for all $t, t^{\prime} \geq 0, F\left(t, t, t, t^{\prime}, t, t^{\prime}\right) \leq 0$ implies $t \leq h_{2} t^{\prime}$.

In the following examples, property $\left(F_{1}\right)$ is obviously.

Example $10 F\left(t_{1}, \ldots, t_{6}\right)=t_{1}-a_{1} t_{2}-a_{2} t_{3}-a_{3} t_{4}-a_{4} t_{5}-a_{5} t_{6}$, where $a_{1}, \ldots, a_{5} \geq 0, a_{3}$ or $a_{5} \neq 0$ and $a_{1}+a_{2}+a_{3}+2 a_{4}+a_{5}<1$.

$\left(\mathrm{F}_{2}\right): \quad$ Let $u, v \geq 0$ and $F(u, v, v, u, u+v, u)=u-a_{1} v-a_{2} v-a_{3} u-a_{4}(u+v)-a_{5} u \leq 0$, which implies $u \leq h_{1} v$, where $0 \leq h_{1}=\frac{a_{1}+a_{2}+a_{4}}{1-\left(a_{3}+a_{4}+a_{5}\right)}<1$.

$\left(\mathrm{F}_{3}\right)$ : Let $t, t^{\prime} \geq 0$ and $F\left(t, t, t, t^{\prime}, t, t^{\prime}\right)=t-a_{1} t-a_{2} t-a_{3} t^{\prime}-a_{4} t-a_{5} t^{\prime} \leq 0$, which implies $t \leq h_{2} t^{\prime}$, where $0<h_{2}=\frac{a_{3}+a_{5}}{1-\left(a_{1}+a_{2}+a_{4}\right)}<1$.

Example $11 F\left(t_{1}, \ldots, t_{6}\right)=t_{1}-k \max \left\{t_{2}, t_{3}, t_{4}, t_{5}, t_{6}\right\}$, where $k \in\left[0, \frac{1}{2}\right)$.

$\left(\mathrm{F}_{2}\right): \quad$ Let $u, v \geq 0$ and $F(u, v, v, u, u+v, u)=u-k \max \{u, v, u+v\}=u-k(u+v) \leq 0$, which implies $u \leq h_{1} v$, where $0 \leq h_{1}=\frac{k}{1-k}<1$.

$\left(\mathrm{F}_{3}\right)$ : Let $t, t^{\prime} \geq 0$ and $F\left(t, t, t, t^{\prime}, t, t^{\prime}\right)=t-k \max \left\{t, t^{\prime}\right\} \leq 0$. If $t>t^{\prime}$, then $t(1-k) \leq 0$, a contradiction. Hence, $t \leq t^{\prime}$, which implies $t \leq h_{2} t^{\prime}$, where $0<h_{2}=k<1$.

In the following, the proofs are similar and thus omitted.

Example $12 F\left(t_{1}, \ldots, t_{6}\right)=t_{1}^{2}-t_{1}\left(a t_{2}+b t_{3}+c t_{4}\right)-d t_{5} t_{6}$, where $a, b, c, d \geq 0$ and $a+b+c+2 d<1$

Example $13 F\left(t_{1}, \ldots, t_{6}\right)=t_{1}^{2}-a \max \left\{t_{2}^{2}, t_{3}^{2}, t_{4}^{2}\right\}-b t_{5} t_{6}$, where $a, b \geq 0$ and $a+2 b<1$.

Example $14 F\left(t_{1}, \ldots, t_{6}\right)=t_{1}^{3}-a t_{1}^{2} t_{2}-b t_{1} t_{2}^{2}-c t_{2} t_{3} t_{4}-d t_{1} t_{5} t_{6}$, where $a, b \geq 0, c, d>0$ and $a+b+c+2 d<1$.

Example $15 F\left(t_{1}, \ldots, t_{6}\right)=t_{1}-a t_{2}-b t_{3}-c \max \left\{2 t_{4}, t_{5}+t_{6}\right\}$, where $a>0, b, c \geq 0$ and $a+b+3 c<1$. 
Example $16 F\left(t_{1}, \ldots, t_{6}\right)=t_{1}^{2}+\frac{t_{1}}{1+t_{5}+t_{6}}-\left(a t_{2}^{2}+b t_{3}^{2}+c t_{4}^{2}\right)$, where $a, b \geq 0, c>0$ and $a+b+c<1$.

\section{MAIN RESULTS}

Theorem 17 Let $(X, G p)$ be a $G p$ - metric space and $T: X \rightarrow X$ be a mapping such that: $F(G p(T x, T x, T y), G p(x, x, y), G p(x, x, T x), G p(y, y, T y), G p(x, x, T y), G p(y, y, T x)) \leq 0$ for all $x, y \in X$, where $F$ satisfy property $\left(\mathrm{F}_{3}\right)$. Then, $T$ has at most a fixed point.

Proof. Suppose that $T$ has two distinct fixed points $u$ and $v$. By (1) we have successively $F(G p(T u, T u, T v), G p(u, u, v), G p(u, u, T u), G p(v, v, T v), G p(u, u, T v), G p(v, v, T u)) \leq 0$,

$F(G p(u, u, v), G p(u, u, v), G p(u, u, u), G p(v, v, v), G p(u, u, v), G p(v, v, u)) \leq 0$.

By $\left(G p_{2}\right)$,

$$
\begin{aligned}
& G p(u, u, u) \leq G p(u, u, v), \\
& G p(v, v, v) \leq G p(v, v, u) .
\end{aligned}
$$

Then, by $\left(F_{1}\right)$ we obtain

$$
F(G p(u, u, v), G p(u, u, v), G p(u, u, v), G p(v, v, u), G p(u, u, v), G p(v, v, u)) \leq 0,
$$

which implies by $\left(F_{3}\right)$ that

$$
G p(u, u, v) \leq h_{2} G p(v, v, u) .
$$

Similarly, by (1) we obtain

$$
G p(v, v, u) \leq h_{2} G p(u, u, v) .
$$

Hence,

$$
G p(u, u, v) \leq h_{2}^{2} G p(u, u, v)
$$

which implies

$$
G p(u, u, v)\left(1-h_{2}^{2}\right) \leq 0 .
$$

Then,

and by Lemma 5 ,

$$
G p(u, u, v)=0
$$

$$
u=v .
$$

Theorem 18 Let $(X, G p)$ be a $G p$-complete metric space and let $T: X \rightarrow X$ satisfying inequality (1), for all $x, y \in X$, where $F \in \mathscr{F}_{G p}$. Then, $T$ has an unique fixed point.

Proof. From (1), for $y=T x$, we get

$$
F\left(G p\left(T x, T x, T^{2} x\right), G p(x, x, T x), G p(x, x, T x), G p\left(T x, T x, T^{2} x\right), G p\left(x, x, T^{2} x\right), G p(T x, T x, T x)\right) \leq 0 \text {. }
$$

By $\left(G p_{4}\right)$, we have

$$
\left.G p\left(x, x, T^{2} x\right) \leq G p(x, x, T x)+G p\left(T x, T x, T^{2} x\right)\right)
$$

and by $\left(G p_{2}\right)$, we have

$$
\left.G p(T x, T x, T x) \leq G p\left(T x, T x, T^{2} x\right)\right) .
$$

Then by $\left(F_{1}\right)$ we obtain

$$
F\left(\begin{array}{c}
G p\left(T x, T x, T^{2} x\right), G p(x, x, T x), G p(x, x, T x), G p\left(T x, T x, T^{2} x\right), \\
G p(x, x, T x)+G p\left(T x, T x, T^{2} x\right), G p\left(T x, T x, T^{2} x\right)
\end{array}\right) \leq 0,
$$

which implies by $\left(F_{2}\right)$ that

$$
G p\left(T x, T x, T^{2} x\right) \leq h_{1} G p(x, x, T x) .
$$


Let $x_{0} \in X$ be an arbitrary point and $x_{n}=T x_{n-1}, n=1,2, \ldots$.

If $x_{n}=x_{n-1}$, then $x_{n}$ is a fixed point of $T$. Suppose that $x_{n} \neq x_{n-1}$, for each $n \in \mathbb{N}$. Then by (15) we obtain

$$
G p\left(T x_{n-1}, T x_{n-1}, T x_{n}\right) \leq h_{1} G p\left(x_{n-1}, x_{n-1}, T x_{n-1}\right),
$$

i.e.,

which implies

$$
G p\left(x_{n}, x_{n}, x_{n+1}\right) \leq h_{1} G p\left(x_{n-1}, x_{n-1}, T x_{n-1}\right),
$$

$$
G p\left(x_{n}, x_{n}, x_{n+1}\right) \leq h_{1}^{n} G p\left(x_{0}, x_{0}, x_{1}\right) .
$$

Moreover, for $m, n \in \mathbb{N}, m>n$, from repeating use of $\left(G p_{4}\right)$ we obtain

$$
G p\left(x_{n}, x_{n}, x_{m}\right) \leq \sum_{j=n}^{m-1} h_{1}^{j} G p\left(x_{0}, x_{0}, x_{1}\right) \leq \frac{h_{1}^{n}}{1-h_{1}^{n}} G p\left(x_{0}, x_{0}, x_{1}\right) .
$$

By Lemma 7,

$$
G p\left(x_{n}, x_{n}, x_{m}\right) \leq 2 G p\left(x_{n}, x_{n}, x_{m}\right) \leq \frac{h_{1}^{n}}{1-h_{1}^{n}} G p\left(x_{0}, x_{0}, x_{1}\right) .
$$

Consequently,

$$
\lim _{n, m \rightarrow \infty} G p\left(x_{n}, x_{n}, x_{m}\right)=0
$$

and thus, $\left\{x_{n}\right\}$ is a $G p$ - Cauchy sequence. Since $(X, G p)$ is a $G p$ - complete metric space and by Theorem 3, there exists $z \in X$ such that

$$
\lim _{n, m \rightarrow \infty} G p\left(x_{n}, x_{n}, x_{m}\right)=\lim _{n \rightarrow \infty} G p\left(z, x_{n}, x_{n}\right)=G p(z, z, z)=0
$$

we prove that $z$ is a fixed point of $T$.

By (1) we have successively

$$
\begin{aligned}
& F\left(G p\left(T x_{n}, T x_{n}, T z\right), G p\left(x_{n}, x_{n}, z\right), G p\left(x_{n}, x_{n}, T x_{n}\right), G p(z, z, T z), G p\left(x_{n}, x_{n}, T z\right), G p\left(z, z, T x_{n}\right)\right) \leq 0, \\
& F\left(G p\left(x_{n+1}, x_{n+1}, T z\right), G p\left(x_{n}, x_{n}, z\right), G p\left(x_{n}, x_{n}, x_{n+1}\right), G p(z, z, T z), G p\left(x_{n}, x_{n}, T z\right), G p\left(z, z, x_{n+1}\right)\right) \leq 0 .
\end{aligned}
$$

By Lemmas 8 and 9, Theorem 3 and (22), letting $n$ tends to infinity we obtain

$$
F(G p(z, z, T z), 0,0, G p(z, z, T z), G p(z, z, T z), G p(z, z, T z)) \leq 0,
$$

which implies by $\left(F_{2}\right)$ that $G p(z, z, T z)=0$. By Lemma $5, z=T z$. Hence, $z$ is a fixed point of $T$. By Theorem 17, $z$ is the unique fixed point of $T$.

From Theorem 18 and Example 11 we obtain

Corollary 19 Let $(X, G p)$ be a $G p$ - complete metric space and $T: X \rightarrow X$ be a mapping such that

$$
G p(T x, T x, T y) \leq k \max \{G p(x, x, y), G p(x, x, T x), G p(y, y, T y), G p(x, x, T y), G p(y, y, T x)\}
$$

where $k \in\left[0, \frac{1}{2}\right)$, for all $x, y \in X$. Then $T$ has an unique fixed point.

From Theorem 18 and Examples 10, 12-16 we obtain other results.

Example 20 Let $X=[0, \infty)$ and $G p: X^{3} \rightarrow \mathrm{R}$ defined by $G p(x, y, z)=\max \{x, y, z\}$. Then $(X, G p)$ is a Gp - complete metric space. Let $T: X \rightarrow X$ be defined by $T x=\frac{x}{x+3}$. Without loss of generality, we assume that $x \geq y$. Then

$$
G p(T x, T x, T y)=\frac{x}{x+3} \leq \frac{x}{3}=\frac{1}{3} G p(x, x, y) \leq k G p(x, x, y),
$$

where $k \in\left[\frac{1}{3}, \frac{1}{2}\right)$ which implies

$G p(T x, T x, T y) \leq k \max \{G p(x, x, y), G p(x, x, T x), G p(y, y, T y), G p(x, x, T y), G p(y, y, T x)\}$.

By Corollary 19, $T$ has an unique fixed point $x=0$. Moreover, $G p(0,0,0)=0$. 


\section{WELL POSEDNESS PROBLEM OF FIXED POINT IN $G p$ - METRIC SPACES}

Definition 21 ([37]) Let $(X, d)$ be a metric space and $f:(X, d) \rightarrow(X, d)$ be a mapping. The fixed point problem of $f$ is said to be well posed if:

1) $f$ has an unique fixed point $x_{0}$;

2) for any sequence $\left\{x_{n}\right\} \in X$ with $\lim _{n \rightarrow \infty} d\left(x_{n}, f x_{n}\right)=0$ we have $\lim _{n \rightarrow \infty} d\left(x_{n}, x_{0}\right)=0$.

Definition 22 Let $(X, G p)$ be a Gp - metric space and let $T: X \rightarrow X$ be a self mapping. The fixed point problem of $T$ is said to be well posed if:

1) $T$ has an unique fixed point $x_{0}$;

2) for any sequence $\left\{x_{n}\right\} \in X$ with $\lim _{n \rightarrow \infty} G p\left(x_{n}, x_{n}, T x_{n}\right)=0$ we have $\lim _{n \rightarrow \infty} G p\left(x_{0}, x_{0}, x_{n}\right)=0$.

Definition 23 A function $F: \mathbb{R}_{+}^{6} \rightarrow \mathbb{R}$ has property $\left(F_{q}\right)$ if for $u, v, w \geq 0$ and $F(u, v, 0, w, u, v) \leq 0$, there exists $q \in(0,1)$ such that $u \leq q \max \{v, w\}$.

Remark 24 All the functions $F$ from Examples 10 - 16 have property $\left(F_{q}\right)$.

Theorem 25 Let $(X, G p)$ be a Gp - symmetric space and let $T: X \rightarrow X$ be a mapping satisfying the conditions from Theorem 18 and $F$ having property $\left(F_{q}\right)$. Then the fixed point problem of $T$ is well posed.

Proof. By Theorem 18, T has an unique fixed point $x_{0}$. Let $\left\{x_{n}\right\}$ be a sequence in $X$ such that $\lim _{n \rightarrow \infty} G p\left(x_{n}, x_{n}, T x_{n}\right)=0$. By (1) we have successively

$$
F\left(\begin{array}{c}
G p\left(T x_{0}, T x_{0}, T x_{n}\right), G p\left(x_{0}, x_{0}, x_{n}\right), G p\left(x_{0}, x_{0}, T x_{0}\right), \\
G p\left(x_{n}, x_{n}, T x_{n}\right), G p\left(x_{0}, x_{0}, T x_{n}\right), G p\left(x_{n}, x_{n}, T x_{0}\right)
\end{array}\right) \leq 0,
$$

$F\left(G p\left(x_{0}, x_{0}, T x_{n}\right), G p\left(x_{0}, x_{0}, x_{n}\right), 0, G p\left(x_{n}, x_{n}, T x_{n}\right), G p\left(x_{0}, x_{0}, T x_{n}\right), G p\left(x_{n}, x_{n}, x_{0}\right)\right) \leq 0$.

Since $G p$ is symmetric, $G p\left(x_{n}, x_{n}, x_{0}\right)=G p\left(x_{0}, x_{0}, x_{n}\right)$, hence

$F\left(G p\left(x_{0}, x_{0}, T x_{n}\right), G p\left(x_{0}, x_{0}, x_{n}\right), 0, G p\left(x_{n}, x_{n}, T x_{n}\right), G p\left(x_{0}, x_{0}, T x_{n}\right), G p\left(x_{0}, x_{0}, x_{n}\right)\right) \leq 0$.

Since $F$ satisfy property $\left(F_{q}\right)$ we have

$$
\begin{aligned}
G p\left(x_{0}, x_{0}, T x_{n}\right) & \leq q \max \left\{G p\left(x_{0}, x_{0}, x_{n}\right), G p\left(x_{n}, x_{n}, T x_{n}\right)\right\} \\
& \leq q\left[G p\left(x_{0}, x_{0}, x_{n}\right)+G p\left(x_{n}, x_{n}, T x_{n}\right)\right] .
\end{aligned}
$$

By $\left(G p_{4}\right)$,

$$
\begin{aligned}
G p\left(x_{0}, x_{0}, x_{n}\right) & \left.\leq G p\left(x_{0}, T x_{n}, T x_{n}\right)+G p\left(T x_{n}, x_{0}, x_{0}\right)\right\} \\
& \leq G p\left(x_{n}, T x_{n}, T x_{n}\right)+q\left[G p\left(x_{0}, x_{0}, T x_{n}\right)+G p\left(x_{n}, x_{n}, T x_{n}\right)\right],
\end{aligned}
$$

which implies

$$
G p\left(x_{0}, x_{0}, x_{n}\right) \leq \frac{1+q}{1-q} G p\left(x_{n}, x_{n}, T x_{n}\right) .
$$

Hence,

$$
\lim _{n \rightarrow \infty} G p\left(x_{0}, x_{0}, x_{n}\right)=0
$$

and the fixed point problem of $T$ is well posed.

Corollary 26 Let $(X, G p)$ be a $G p$ - symmetric space and let $T: X \rightarrow X$ be a function satisfying the conditions of Corollary 19. Then, the fixed point problem of $T$ is well posed. 


\section{References}

[1] Abbas T., Nazir T. and Radenović S., Some periodic point results in generalized metric spaces, Appl. Math. Comput., 217, 4084 - 4099 (2010).

[2] Abdeljawad T., Karapinar E. and Tas K., Existence and uniqueness of common fixed points on partial metric spaces, Appl. Math. Lett., 24 (11), 1894 - 1899 (2011).

[3] Akkouchi M. and Popa V., Well posedness of common fixed point problem for three mappings under strict contractive conditions, Bul. Univ. Petrol-Gaze Ploieşti. Ser. Mat. Inform. Fiz., 61 (2), 1 - 10 (2009).

[4] Akkouchi M. and Popa V., Well posedness of a fixed point problem using $G$ - functions, Sci. Stud. Res. Ser. Math. Inform., 20, 5 - 12 (2010).

[5] Akkouchi M. and Popa V., Well posedness of a fixed point problem for mappings satisfying an implicit relation, Demonstr. Math., 43 (4), 923 - 929 (2010).

[6] Akkouchi M. and Popa V., Well posedness of fixed point problem for hybrid pairs of mappings, Fasc. Math., 46, 5 - 16 (2011).

[7] Altun I., Sola F. and Simsek H., Generalized contractive principle on partial metric spaces, Topology Appl., 157 (18), 2778 - 2785 (2010).

[8] Aydi H., Karapinar E. and Salimi P., Some fixed point results in $G p$ - metric spaces, Int. J. Appl. Math., Article ID 891713 (2012).

[9] Barakat M. A. and Zidad A. M., A common fixed point theorem for weak contractive maps in $G p$ - metric spaces, J. Egyptian Math. Soc. DOI: 10.1016/j.joems.2014.06.008 (2014).

[10] Bilgili N., Karapinar E. and Salimi P., Fixed point theorems for generalized contractions on $G p$ metric spaces, J. Ineq. Appl. 2013:39 (2013).

[11] Chi R., Karapinar E. and Thanh T. D., A generalized contraction principle in partial metric spaces, Math. Comput. Modelling, 53, 1673 - 1681 (2012).

[12] De Blasi F. S. and Myjak J., Sur la porosité de l'ensemble des contractions sans point fixe, Comptes Rend. Acad. Sci. Paris, 308, 51 - 54 (1989).

[13] Dhage B. C., Generalized metric spaces and mappings with fixed point, Bull. Calcutta Math. Soc., 8, 329 - 336 (1992).

[14] Dhage B. C., Generalized metric spaces and topological structures, I, An. Ştiinţ. Univ. Al. I. Cuza Iaşi. Mat., 46, 3 - 24 (2000).

[15] Kadelburg Z., Nashine H. K. and Radanović S., Fixed point results under various contractive conditions in partial metric spaces, RACSAM, 10, 241 - 256 (2013).

[16] Karapinar E. and Erhan I. M., Fixed point theorems for operators on partial metric spaces, Appl. Math. Lett., 24 (11), 1900 - 1904 (2011).

[17] Kaushal D. S. and Pagey S. S., Some results of fixed point theorems on complete $G$ - metric spaces, South Asian J. Math., 2 (4), 318 - 324 (2014).

[18] Lahiri B. K. and Das P., Well posedness and porosity of certain classes of operators, Demonstr. Math., 38, 170 - 176 (2005).

[19] Matthews S., Partial metric topology and applications, Proc. 8th Summer Conference on General Topology and Applications, Ann. New York Acad. Sci., 728, 183 - 197 (1994).

[20] Mohanta S. K., Some fixed point theorems in $G$ - metric spaces, An. Ştiinţ. Univ. Ovidius Constanţa. Ser. Mat., 20 (1), 285 - 306 (2012).

[21] Mustafa Z. and Sims B., Some remarks concerning $D$ - metric spaces, Conf. Fixed Point Theory Appl., Yokohama, 184 - 198 (2004).

[22] Mustafa Z. and Sims B., A new approach to generalized metric spaces, J. Nonlinear Convex Anal., 7 (2), 289 - 297 (2006).

[23] Mustafa Z., Obiedat H. and Awadeh F., Some fixed point theorem for mappings on complete $G$ metric spaces, Fixed Point Theory Appl., Article ID 189870 (2008).

[24] Mustafa Z. and Sims B., Fixed point theorems for contractive mappings in complete $G$ - metric spaces, Fixed Point Theory Appl., Article ID 917175 (2009). 
[25] Mustafa Z., Shatanawi W. and Bataineh M., Existence of fixed point results in $G$ - metric spaces, Intern. J. Math. Math. Sci., Article ID 283028 (2009).

[26] Mustafa Z. and Obiedat H., A fixed point theorem of Reich in $G$ - metric spaces, Cubo. A Math. J., 12 (1), 83 - 93 (2010).

[27] Mustafa Z., Khandagji M. and Shatanawi W., Fixed point results on complete $G$ - metric spaces, Stud. Sci. Math. Hung., 48 (3), 304 - 319 (2011).

[28] Popa V., Fixed point theorems for implicit contractive mappings, Stud. Cercet. Ştiinț. Ser. Mat., Univ. Bacău, 7, 129 - 133 (1997).

[29] Popa V., Some fixed point theorems for compatible mappings satisfying an implicit relation, Demonstr. Math., 32, 157 - 163 (1999).

[30] Popa V., Well posedness of fixed point problem in orbitally complete metric spaces, Stud. Cercet. Ştiinț. Ser. Mat., Univ. Bacău, 16, Suppl., 209 - 214 (2006).

[31] Popa V., Well posedness of fixed point problem in compact metric spaces, Bul. Univ. PetrolGaze Ploieşti, Ser. Mat. Inform. Fiz., 60 (1), 1 - 4 (2008).

[32] Popa V., A general fixed point theorem for several mappings in $G$ - metric spaces, Sci. Stud. Res., Ser. Math. Inform., 21 (1), 205 - 214 (2011).

[33] Popa V. and Patriciu A.-M., Two general fixed point theorems for pairs of weakly compatible mappings in complete $G$ - metric spaces, Novi Sad J. Math., 42 (2), 49 - 60 (2012).

[34] Popa V. and Patriciu A.-M., A general fixed point theorem for mappings satisfying an $\square$ implicit relation in complete $G$ - metric spaces, Gazi Univ. J. Sci., 25 (2), 403 - 408 (2012).

[35] Popa V. and Patriciu A.-M., A general fixed point theorem for pair of weakly compatible mappings in $G$ - metric spaces, J. Nonlinear Sci. Appl., 5 (2), 151 - 160 (2012).

[36] Popa V. and Patriciu A.-M., Fixed point theorems for mappings satisfying an implicit relation in complete $G$ - metric spaces, Bul. Inst. Politehn. Iaşi, Ser. Mat. Mec. Teor. Fiz., 59 (63), 97 - 123 (2013).

[37] Reich S. and Zaslavski A. J., Well posedness of fixed point problem, Far East J. Math. Sci., Special Volume, Part III, 393 - 401 (2001).

[38] Shatanawi W., Fixed point theory for contractive mappings satisfying $\square$ - maps in $G$ - metric spaces, Fixed Point Theory Appl., Article ID 181650 (2010).

[39] Shatanawi W., Common fixed point results for two self - mappings in $G$ - metric spaces, Mat. Vesn., 65 (2), 143 - 150 (2013).

[40] Shatanawi W. and Postolache M., Some fixed point results for a $G$ - weak contraction in $G$ metric spaces, Abstr. Appl. Anal., Article ID 815870 (2012).

[41] Shatanawi W., Chauhan S., Postolache M., Abbas N. and Radenović S., Common fixed point for contractive mappings in $G$ - metric spaces, J. Adv. Math. Stud., 6 (1), 53 - 72 (2013).

[42] Srivastava R., Agrawal S., Bhardwaj R. and Vardava R., Fixed point theorems in complete $G$ metric spaces, South Asian J. Math., 2 (2), 167 - 174 (2013).

[43] Vats R. K., Kumar S. and Siharg V., Fixed point theorems in complete $G$ - metric spaces, Fasc. Math., 47, 127 - 139 (2011).

[44] Vetro C. and Vetro F., Common fixed points of mappings satisfying implicit relations in partial metric spaces, J. Nonlinear Sci. Appl., 6, 152 - 160 (2013).

[45] Zand M. R. A. and Nezhad A. N., A generalization of partial metric spaces. J. Contemporary Appl. Math. 1(2011), no. 1, 86-93. 